\section{Diferenciais dos aglomerados de nascidos vivos no Município de São Paulo, Brasil, 2010}

\author{
Differences in live birth clusters in the city of \\ São Paulo, Brazil, 2010
}

Diferenciales de los aglomerados de nacidos

vivos en el municipio de São Paulo, Brasil, 2010
Patricia Carla dos Santos 1

Zilda Pereira da Silva 1

Francisco Chiaravalloti Neto 1

Gizelton Pereira Alencar 1

Marcia Furquim de Almeida 1

\section{Resumo}

O objetivo do estudo foi identificar aglomerados de nascimentos segundo o tipo de hospital (SUS e não SUS) e a existência de diferenciais quanto a características socioeconômicas, materno-infantis e de acesso, medidos pela distância entre as residências maternas e os hospitais onde se deram os partos. Os nascimentos ocorridos de mães residentes no Município de São Paulo, Brasil, em 2010 foram georreferenciados e alocados nas 310 áreas de ponderação do censo demográfico, além de classificados segundo hospital de nascimento (SUS e não SUS). Foram identificados aglomerados espaciais por meio da técnica de varredura espacial para dependência espacial dos nascimentos SUS e não SUS, com a formação de dez aglomerados SUS e sete não SUS. Os nascimentos em hospitais não SUS formaram aglomerados situados na área central, onde há menor proporção de domicílios de baixa renda. Os aglomerados de nascidos vivos SUS localizaram-se nas bordas da cidade, onde são mais frequentes domicílios em aglomerados subnormais. Os aglomerados tanto SUS como não SUS não são homogêneos entre si, visto que há diferenças em relação a idade das mães, escolaridade, número de consultas de pré-natal e recémnascidos muito prematuros. A distância média teórica percorrida pelas mães até o hospital foi 51,8\% menor nos aglomerados SUS $(5,1 \mathrm{~km})$ que nos não SUS $(9,8 \mathrm{~km})$. A formação de aglomerados de nascimentos mostrou diferenciais das características maternas, gestação, parto e recém-nascidos, além de ter apresentado distribuição espacial radial-concêntrica, refletindo os diferenciais socioeconômicos existentes na cidade. A menor distância nos nascimentos SUS indica a regionalização da assistência ao parto no Município de São Paulo.

\section{Correspondência}

P. C. Santos

Faculdade de Saúde Pública, Universidade de São Paulo. Av. Dr. Arnaldo 715, São Paulo, SP 01246-904, Brasil. patcarla@usp.br

${ }^{1}$ Faculdade de Saúde Pública, Universidade de São Paulo, São Paulo, Brasil.

Nascimento Vivo; Disparidades nos Níveis de Saúde; Sistemas de

Informação Geográfica; Hospitais Públicos; Hospitais Privados 


\section{Introdução}

Um evento de saúde não é somente uma manifestação do indivíduo, mas é também uma expressão das condições sociais do lugar. Assim, espaço e população tornam-se uma unidade que expressa os determinantes sociais, os problemas de saúde e o desenvolvimento de ações de promoção de equidade 1.

Estudos de base populacional podem fornecer informações essenciais para intervenções de saúde pública ao estudar associações entre disparidades socioeconômicas e desfechos materno-infantis. Para tanto, é imprescindível que, além da disponibilidade de estatísticas vitais e de dados socioeconômicos, também haja ferramentas disponíveis para que essas informações possam ser úteis para melhor compreensão dos fenômenos e intervenções eficazes 2 .

Uma dessas ferramentas, o georreferenciamento, é utilizado como instrumento analítico, partindo da hipótese de que as informações individualizadas podem vir a formar agregados espaciais. Esses agregados não se constituem apenas no somatório dos indivíduos que os compõem, mas expressam a interação existente entre os indivíduos que formam esses agrupamentos, os quais compartilham entre si valores, comportamentos, características socioeconômicas e o espaço 3 .

As técnicas de georreferenciamento trouxeram um avanço aos estudos de diferenciais inter e intraurbanos de mortalidade 1,4,5, de nascimentos 6,7,8,9,10,11 e de acesso aos serviços de saúde 12,13, pois permitiram visualizar as relações complexas que ocorrem na ocupação do solo urbano. Muitos desses estudos utilizam áreas quentes 11,14 para identificar a localização socioespacial dos eventos, porém o emprego de técnicas de varredura espacial 15, que permitem identificar a formação de clusters ou aglomerados, pode propiciar melhor identificação das características da distribuição espacial dos eventos.

Um dos aspectos relacionados à ocupação urbana é a mobilidade. O acesso geográfico é uma medida da oferta de serviços de saúde. Em relação ao parto, se os serviços de saúde não estão igualmente distribuídos dentro de uma região, isso pode tornar-se vulnerabilidade para a mãe e para o concepto 12 . Dessa forma, a formação socioespacial evidencia as desigualdades sociais entre segmentos populacionais no espaço interurbano 16 . Ainda que a distância até os serviços de saúde represente apenas uma das dimensões do acesso 17 , a distância entre o hospital de nascimento e a residência materna tem sido empregada também como uma medida socioeconômica 13.

Há alguns critérios para a escolha da unidade espacial: disponibilidade e qualidade das informações, reconhecimento da unidade por parte da população, existência de grupos populacionais organizados e de instâncias administrativas do Estado na unidade e permissão para níveis progressivos 18 de agregação. A observação das condições de vida e saúde em microáreas permite identificar melhor a heterogeneidade de condições de vida. A despeito da diversidade na disponibilidade de dados em vários níveis de agregação, a escolha da unidade de análise deve ser influenciada pelo interesse em avaliar a situação de saúde em uma determinada localidade 19.

Estudos anteriores mostraram diferenças na distribuição espacial das características socioeconômicas, maternas, da gestação e dos recém-nascidos nos hospitais SUS e não SUS e também a existência de um padrão de distribuição espacial de nascimentos 6,11,13. Sendo assim, o objetivo do estudo é identificar aglomerados de nascimentos segundo tipo de hospital (SUS e não SUS) e a existência de diferenciais quanto a características socioeconômicas, materno-infantis e de acesso, medidos pela distância entre a residência materna e os hospitais onde se deram os nascimentos.

\section{Material e métodos}

Foi realizado um estudo ecológico cuja população é o conjunto de nascidos vivos hospitalares de mães residentes, ocorridos no Município de São Paulo, Brasil, no período de 1o de janeiro de 2010 a 31 de dezembro de 2010. Os dados são provenientes do Sistema de Informações sobre Nascidos Vivos (SINASC) e foram obtidos da Secretaria de Estado da Saúde de São Paulo.

Os nascidos vivos foram classificados segundo o tipo de hospital onde foi realizado o parto. Com base nas informações do Cadastro Nacional de Estabelecimentos de Saúde (CNES), foram considerados como hospitais SUS os públicos (exclusivamente SUS), os privados e os filantrópicos que atendem à população usuária do SUS e a uma parcela menor de clientela privada. Os hospitais não SUS, por sua vez, são os hospitais privados que atendem exclusivamente a clientela particular e com plano de 
saúde. A partir de georreferenciamento dos endereços dos hospitais, obteve-se a distribuição espacial dessas unidades de saúde.

Os endereços de residência das mães registrados nas declarações de nascidos vivos também foram georreferenciados, e as coordenadas geográficas foram alocadas segundo Áreas de Ponderação (AP). Essas unidades geográficas são formadas por agrupamentos de setores censitários contíguos, para os quais se podem calcular estimativas baseadas nas informações do questionário da amostra do Censo Demográfico de 2010 (Instituto Brasileiro de Geografia e Estatística; http://www.ibge.gov.br). O Município de São Paulo é dividido em 310 áreas de ponderação.

Para a obtenção dos aglomerados de nascimentos, foi empregada a técnica de varredura espacial, utilizando-se o software SaTScan 9.4 (http://www.satscan.org). A estatística de varredura espacial é definida por uma janela geográfica circular que se move pela área de interesse, em que o círculo é capaz de incluir diferentes conjuntos de áreas vizinhas, sob a hipótese nula de que não há formação de aglomerados 15 . O número de eventos em cada área é considerado como distribuído de acordo com a população sob risco conhecida que, neste estudo, foi considerada como a população feminina em idade fértil (10 a 49 anos), e a hipótese nula é a de que o número esperado de casos em cada área seja proporcional ao tamanho de sua população 20. A técnica SatScan emprega um percentual pré-determinado como valor máximo de população de risco para rastrear os conglomerados.

Para identificação dos aglomerados de nascidos vivos em hospitais SUS e não SUS, empregou-se o modelo de distribuição de probabilidade de Poisson. Esse modelo requer a contagem de casos e população para cada região de localização dos eventos, assim como o conhecimento das coordenadas geográficas para cada uma dessas localizações. Foi considerado como população de risco o grupo de mulheres em idade fértil, de 10 a 49 anos, de cada área de ponderação, considerando-se para a varredura que um aglomerado poderia englobar até $50 \%$ da população de mulheres, tanto para hospitais SUS como não SUS. Para avaliar a significância do teste, foram usadas simulações de Monte Carlo. Os maiores valores de razão de verossimilhança para os casos observados e o valor de $\mathrm{p}$ foram calculados para cada aglomerado. Os mapas temáticos foram obtidos utilizando-se o software ArcGIS 10.2 (http://www.esri.com/software/arcgis/index.html).

Os aglomerados foram caracterizados segundo aspectos socioeconômicos das áreas de ponderação onde estavam localizados, por meio das variáveis proporção de domicílios em aglomerados subnormais e proporção de domicílios com renda familiar inferior a dois salários mínimos, obtidas no Censo Demográfico de 2010. Foram também utilizadas variáveis obtidas do SINASC sobre as mães: idade e escolaridade, características da gestação e do parto (número de consultas de pré-natal e tipo de parto), bem como características dos nascidos vivos (peso ao nascer, idade gestacional e escore do Apgar do $5^{\circ}$ minuto). Foram calculadas as distâncias teóricas médias da residência materna ao hospital de nascimento para cada aglomerado. Foi utilizado teste qui-quadrado para testar a diferença entre a proporção encontrada nos nascimentos SUS e não SUS. O teste qui-quadrado também foi utilizado para testar a diferença entre as proporções encontradas em cada aglomerado e a média total de aglomerados SUS e não SUS.

O estudo faz parte do projeto Distribuição Espacial dos Nascimentos, Trajeto das Mães dos Domicílios aos Hospitais de Parto e Mortalidade Neonatal no Município de São Paulo, 2010 (FAPESP 2011/21718-1), aprovado pelo comitê de ética da Faculdade de Saúde Pública da Universidade de São Paulo (COEP 167/11).

\section{Resultados}

Do total de 174.226 nascimentos ocorridos em 83 hospitais do Município de São Paulo em 2010, foram geocodificados 165.428 (95\%) endereços de residência materna. Desses, 98.140 (59,3\%) são de nascidos vivos em 42 hospitais SUS e 67.288 (40,7\%) de nascidos vivos em 41 hospitais não SUS, localizados em sua maioria na região central da cidade (Figura 1). Foram encontradas diferenças estatisticamente significantes entre as características maternas, da gestação e dos nascidos vivos em hospitais SUS e não SUS, exceto para nascimentos pré-termos tardios (32 a 36 semanas de gestação) e para pré-termos precoces no SUS. A distância percorrida pelas mães até o hospital foi 37,8\% menor para o total de nascimentos no SUS $(6,1 \mathrm{~km})$ que aquela percorrida pelas mães do conjunto de nascimentos não SUS $(9,8 \mathrm{~km})$. 


\section{Figura 1}

Aglomerados de nascimentos em hospitais SUS e não SUS. Município de São Paulo, Brasil, 2010.

1a) Aglomerados SUS
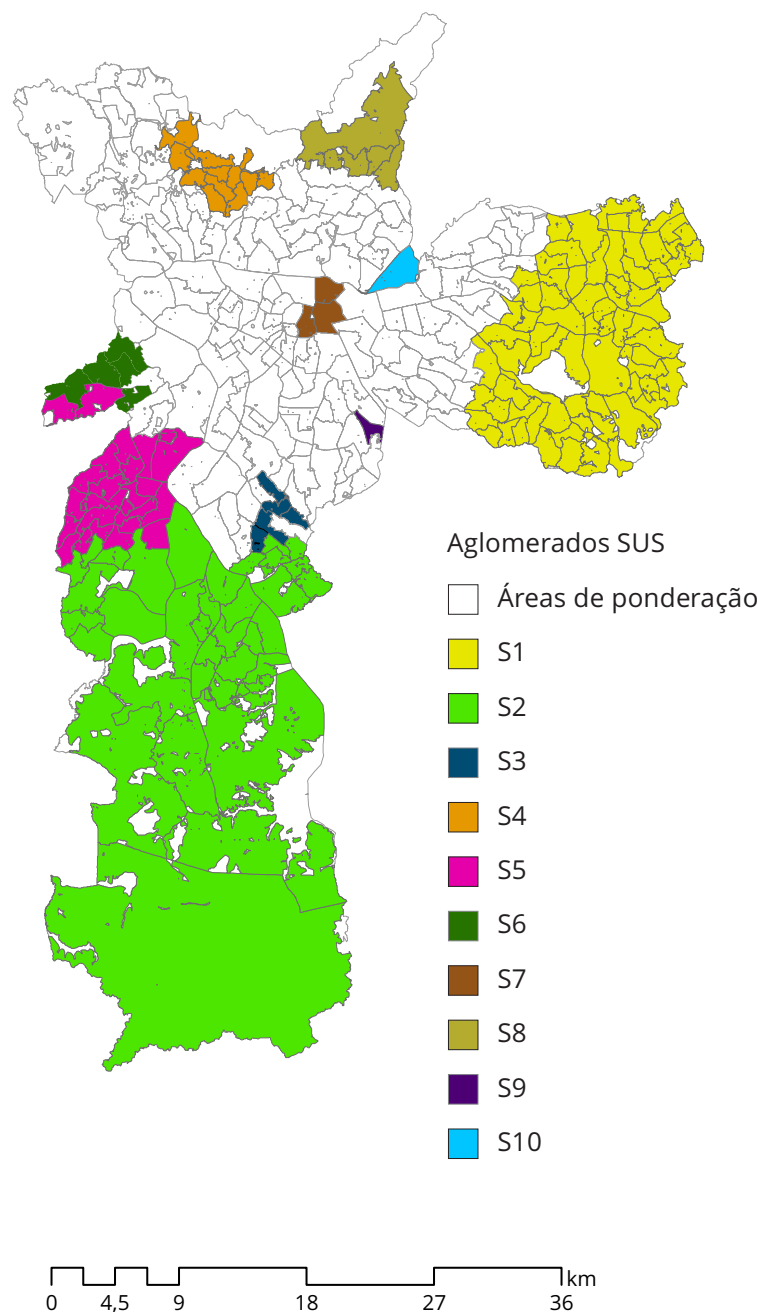

1b) Aglomerados não SUS
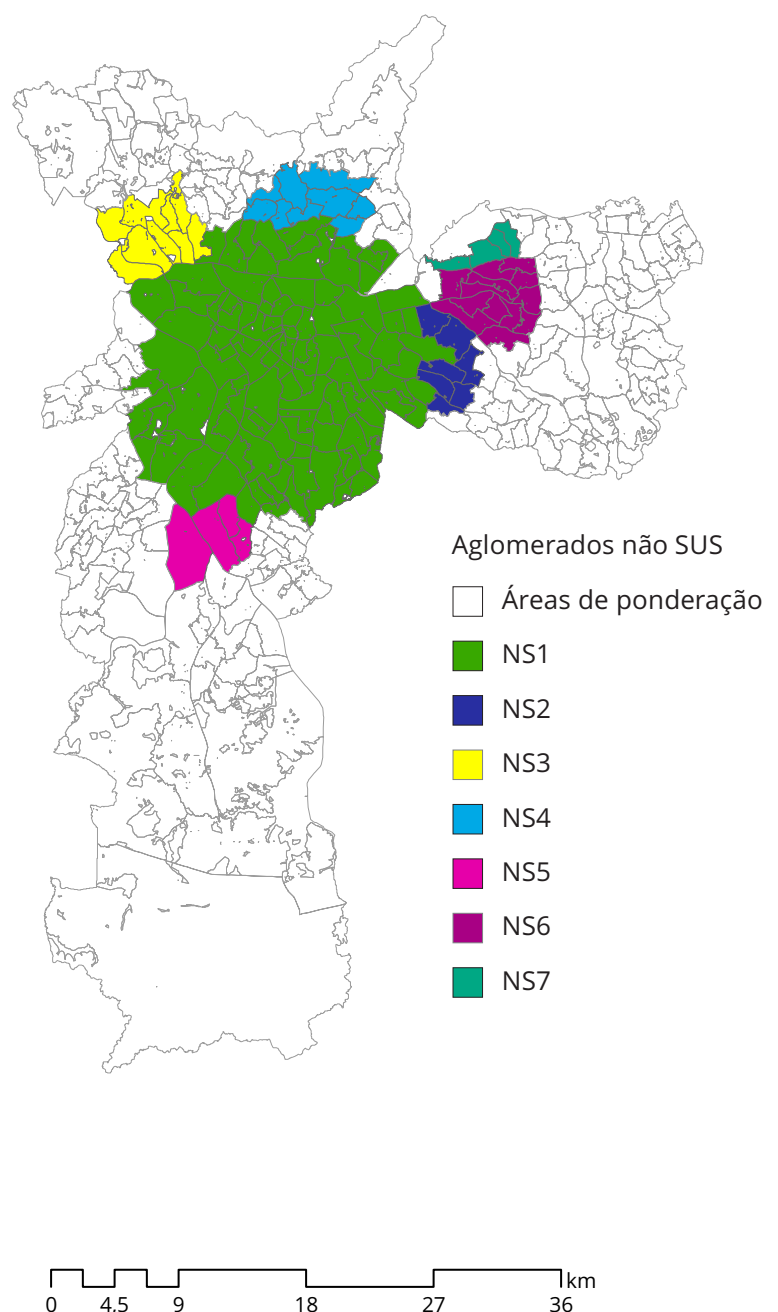

A frequência de mães com < 20 anos foi 4,4 vezes maior nos nascimentos em hospitais SUS do que nos não SUS. Relação inversa foi encontrada com relação à proporção de mães com mais de 35 anos, que foi quase duas vezes maior em hospitais não SUS que no SUS. A frequência de baixa escolaridade materna (< 8 anos de estudo) foi 4,7 vezes maior nos nascimentos em hospitais SUS, se comparada à proporção de mães usuárias não SUS. Em relação ao tipo de parto, a proporção de cesáreas na rede privada foi 2,6 vezes maior do que na rede SUS. A proporção de nascidos vivos com baixo peso ao nascer foi maior nos nascidos em hospitais SUS (Tabela 1).

Foram identificados dez aglomerados significativos ( $<<0,05)$ de nascidos vivos SUS (S1 a S10), representando $39,3 \%$ do total de nascidos vivos e sete aglomerados significativos não SUS (NS1 a NS7), com 37.234 nascidos vivos, 22,5\% do total (Tabela 2). Cerca de $40 \%$ dos nascimentos não mostraram dependência espacial.

A Figura 1 mostra a localização dos aglomerados SUS e não SUS. Os aglomerados de nascidos vivos SUS situam-se predominantemente nas regiões periféricas da cidade: região sul (S2, S3, S5 e S6), 
Tabela 1

Perfil dos nascidos vivos segundo hospital SUS e não SUS. Município de São Paulo, Brasil, 2010.

\begin{tabular}{|c|c|c|c|}
\hline & Total & SUS & Não SUS \\
\hline Nascidos vivos [n (\%)] & $165.428(100,0)$ & $98.140(59,3)$ & $67.288(40,7)$ \\
\hline Distância média (km) & 7,6 & 6,1 & 9,8 \\
\hline$<20$ anos $(\%)$ & 13,1 & 19,0 * & 4,3 * \\
\hline$\geq 35 \operatorname{anos}(\%)$ & 15,5 & 11,3 * & 21,8 * \\
\hline$\leq 8$ anos de estudo $(\%)$ & 17,7 & 26,0 * & $5,5 *$ \\
\hline$<7$ consultas de pré-natal (\%) & 22,5 & 31,4 * & 9,4 * \\
\hline Parto cesáreo (\%) & 53,7 & 32,3 * & 85,4 * \\
\hline Baixo peso ao nascer (\%) & 9,5 & 10,1 * & 8,6 * \\
\hline Idade gestacional < 32 semanas (\%) & 1,4 & 1,6 & $1,1 *$ \\
\hline Idade gestacional 32 a 36 semanas (\%) & 7,7 & 7,7 & 7,8 \\
\hline Apgar 5o minuto < 7 (\%) & 1,0 & 1,2 * & 0,6 * \\
\hline
\end{tabular}

* Diferença significante $(p<0,05)$ entre o conjunto de nascimentos do Município de São Paulo.

Tabela 2

Proporção de domicílios em aglomerados subnormais e de domicílios com renda domiciliar per capita de até dois salários mínimos das áreas de ponderação dos aglomerados de nascidos vivos SUS e não SUS. Município de São Paulo, Brasil, 2010.

\begin{tabular}{|c|c|c|c|c|}
\hline Aglomerados & Nascidos vivos & $\%$ & $\begin{array}{c}\text { Domicílios em aglomerado } \\
\text { subnormal (\%) }\end{array}$ & $\begin{array}{c}\text { Renda domiciliar per capita }<2 \\
\text { salários mínimos (\%) }\end{array}$ \\
\hline \multicolumn{5}{|l|}{ SUS } \\
\hline S1 & 26.240 & 15,9 & 9,8 * & 85,8 * \\
\hline S2 & 14.769 & 8,9 & 25,2 * & 88,3 * \\
\hline S3 & 2.236 & 1,4 & 22,1 * & 80,7 * \\
\hline S4 & 5.589 & 3,4 & 23,1 * & 87,8 * \\
\hline S5 & 9.734 & 5,9 & 23,7 * & 79,3 * \\
\hline S6 & 2.334 & 1,4 & 20,8 * & 64,0 * \\
\hline S7 & 1.189 & 0,7 & 0,9 * & 68,9 * \\
\hline S8 & 1.603 & 1,0 & 18,9 & 86,2 * \\
\hline S9 & 610 & 0,4 & 82,2 * & 91,6 * \\
\hline $\mathrm{S} 10$ & 733 & 0,4 & 26,6 * & 85,6 \\
\hline Total & 65.037 & 39,3 & 17,5 ** & $84,3 * *$ \\
\hline \multicolumn{5}{|l|}{ Não SUS } \\
\hline NS1 & 25.813 & 15,6 & 4,9 & 41,0 * \\
\hline NS2 & 1.768 & 1,1 & 1,3 * & 65,6 * \\
\hline NS3 & 2.274 & 1,4 & 7,7 * & 64,0 * \\
\hline NS4 & 2.419 & 1,5 & 2,1 * & 55,5 * \\
\hline NS5 & 1.207 & 0,7 & 3,8 & 43,0 * \\
\hline NS6 & 2.852 & 1,7 & 3,5 * & 68,3 * \\
\hline NS7 & 901 & 0,5 & 5,7 & 74,7 * \\
\hline Total & 37.234 & 22,5 & 4,6 ** & 47,7 ** \\
\hline
\end{tabular}

* Diferença significante $(p<0,05)$ entre as características socioeconômicas dos aglomerados de nascimentos SUS e não SUS comparado com a média do conjunto de aglomerados de nascimentos em hospitais SUS e não SUS;

** Diferença significante $(p<0,05)$ entre as características socioeconômicas dos aglomerados de nascimentos em hospitais SUS e não SUS. 
um na porção leste (S1), um ao norte (S4, S8 e S10) e um na região central (S7). O conjunto dos aglomerados de nascidos vivos em hospitais não SUS é formado por um aglomerado na região central do Município de São Paulo (NS1), contíguo aos outros aglomerados: dois situados na região norte (NS3 e NS4), três ao leste (NS2, NS6 e NS7) e um ao sul (NS5) (Figura 1).

Nas áreas de ponderação onde estão localizados os aglomerados de nascidos vivos SUS, a proporção de domicílios em aglomerados subnormais é 280\% maior do que nos aglomerados de nascidos vivos não SUS e estatisticamente significantes. Entre os aglomerados de nascidos vivos SUS, destaca-se o S9, onde $82 \%$ dos domicílios estão situados em aglomerados subnormais, região da favela de Heliópolis. O aglomerado S7, que fica na região central da cidade, apresenta situação inversa, com pequena proporção de domicílios subnormais (0,9\%). Entre os aglomerados de nascimentos não SUS, aqueles situados mais nas bordas do grande aglomerado da região central da cidade (NS3 e NS7) apresentam maior proporção de domicílios subnormais (Tabela 2; Figura 1).

A proporção de domicílios com renda familiar < 2 salários mínimos é 76,7\% maior no conjunto dos aglomerados SUS que nos não SUS, sendo estatisticamente significante. Mais uma vez destacase o aglomerado S9, onde 91,6\% dos domicílios apresentavam renda inferior a 2 salários mínimos (Tabela 2). Entre os aglomerados de nascimentos não SUS, verifica-se que os mais distantes da região central da cidade (NS6 e NS7) apresentam as maiores proporções de baixa renda. Por outro lado, os aglomerados NS1 e NS5 apresentam as menores proporções.

Todas as características maternas, da gestação, do parto e do recém-nascido mostraram diferenças estatisticamente significantes entre os valores médios dos aglomerados SUS e não SUS. Com relação à idade materna, nos aglomerados SUS, a proporção de nascimentos de mães adolescentes é 34,7\% superior ao encontrado nos aglomerados não SUS. Relação inversa é encontrada para os nascimentos de mães de 35 anos e mais, que é 95,1\% maior entre os nascimentos dos aglomerados não SUS. No entanto, destaca-se a elevada participação de nascidos vivos de mães adolescentes nos aglomerados não SUS NS3, NS6 e NS7, que se situam nas bordas do grande aglomerado central, apresentando perfil mais próximo daquele encontrado nos aglomerados SUS. O aglomerado NS1 na região central da cidade apresentou a menor proporção de mães adolescentes (14,7\%) (Tabela 3).

Os aglomerados de nascimentos SUS apresentam uma proporção 167\% maior de mães de baixa escolaridade (< 8 anos de estudo) do que no conjunto dos aglomerados não SUS. Os aglomerados S1, S9 e S10 apresentaram proporção superior a 10\% de mães com baixa escolaridade.

Cerca de 30\% das mães residentes nos aglomerados de nascimentos SUS não tinham realizado sete consultas de pré-natal, ao passo que, para as mães dos aglomerados não SUS, essa proporção foi quatro vezes menor (7,2\%). Com exceção do aglomerado S3, todos os demais, cujas mães tiveram menos que sete consultas, apresentaram proporção elevada de nascimentos. Entre os aglomerados não SUS, destacam-se três aglomerados (NS7, NS4 e NS3) em que mais de 10\% das mães haviam realizado menos de sete consultas (Tabela 3).

Os aglomerados não SUS apresentaram proporção de nascimentos por cesárea 176\% superior àquela apresentada pelos aglomerados SUS. Verificou-se que há uma pequena amplitude da proporção de nascimentos por cesárea nos aglomerados SUS (26,6\% a 37,5\%). A proporção de cesáreas dentre os nascidos vivos dos aglomerados não SUS é superior a 80\%, atingindo 90,5\% no aglomerado NS2.

A proporção de baixo peso ao nascer foi mais homogênea entre os aglomerados SUS, em que apenas S3 e S8 apresentam valores estatisticamente diferentes do valor médio dos aglomerados. Comportamento semelhante foi observado nos aglomerados não SUS, em que apenas o aglomerado NS3 apresentou proporção estatisticamente diferente do valor médio dos aglomerados não SUS.

A proporção de nascimentos muito pré-termos (< 32 semanas de gestação) registrou homogeneidade entre os aglomerados SUS, com exceção do S7, que apresentou proporção significativamente mais elevada que o conjunto de aglomerados. Com relação ao índice de Apgar no 5o minuto $<7$, a frequência entre os nascimentos dos aglomerados SUS foi o dobro daquela dos nascimentos dos aglomerados não SUS (Tabela 3).

A distância entre a residência materna e o hospital de nascimento nos aglomerados SUS $(5,1 \mathrm{~km})$ foi 51,8\% menor do que nos aglomerados não SUS (9,8km). Os aglomerados não SUS apresentaram maior variação das distâncias, sendo que as mães residentes no grande aglomerado central percorreram distâncias menores $(6 \mathrm{~km})$ que aquelas residentes nos aglomerados localizados mais distantes da região central da cidade, NS6 e NS7 (cerca de $12 \mathrm{~km}$ ). 
Tabela 3

Perfil dos nascidos vivos segundo aglomerados SUS e não SUS. Município de São Paulo, Brasil, 2010.

\begin{tabular}{|c|c|c|c|c|c|c|c|c|c|c|}
\hline \multirow[t]{2}{*}{ Aglomerados } & \multicolumn{4}{|c|}{ Características maternas } & \multicolumn{6}{|c|}{ Características da gestação e do recém-nascido } \\
\hline & $\begin{array}{l}\text { Distância } \\
\text { média (km) }\end{array}$ & $\begin{array}{c}<20 \text { anos } \\
(\%)\end{array}$ & $\begin{array}{c}\geq 35 \text { anos } \\
(\%)\end{array}$ & $\begin{array}{c}<8 \text { anos } \\
\text { de estudo } \\
(\%)\end{array}$ & $\begin{array}{c}<7 \text { consultas } \\
\text { de pré-natal } \\
(\%)\end{array}$ & $\begin{array}{c}\text { Parto } \\
\text { cesáreo } \\
\text { (\%) }\end{array}$ & $\begin{array}{c}\text { Baixo } \\
\text { peso }(\%)\end{array}$ & $\begin{array}{c}<32 \\
\text { semanas } \\
(\%)\end{array}$ & $\begin{array}{c}32 \text { a } 36 \\
\text { semanas } \\
(\%)\end{array}$ & $\begin{array}{c}\text { Apgar 5o } \\
\text { minuto } \\
<7(\%)\end{array}$ \\
\hline \multicolumn{11}{|l|}{ SUS } \\
\hline S1 & 5,8 & 22,7 & 17,6 * & 10,0 * & 29,2 * & 29,0 * & 10,2 & 1,7 & 7,5 & 1,3 \\
\hline S2 & 8,0 & 23,6 * & 17,3 * & 8,1 * & 33,5 * & $32,7 *$ & 10,2 & 1,6 & 7,8 & 1,1 \\
\hline S3 & 5,7 & 22,2 & 17,7 & 8,4 & 31,8 & 30,0 & 11,7 * & 1,6 & 8,6 & 1,4 \\
\hline S4 & 4,2 & 23,6 & 15,3 * & 9,1 & 30,9 & 29,7 * & 10,2 & 1,5 & 7,7 & 1,0 \\
\hline S5 & 6,2 & 21,3 * & 23,6 * & 7,4 * & 21,4 * & 35,0 * & 10,1 & 1,5 & 7,2 & 1,2 \\
\hline S6 & 3,6 & 19,2 * & 22,5 * & 4,3 * & 32,1 * & 37,5 * & 10,1 & 1,7 & 8,5 & 1,7 \\
\hline S7 & 4,9 & 17,7 * & 19,3 & 7,5 & 44,5 * & 30,2 & 9,1 & 2,4 * & 7,4 & 1,8 \\
\hline S8 & 5,6 & 21,1 & 18,7 & 7,4 & 44,4 * & 36,4 * & 12,8 * & 2,1 & 9,4 * & 1,7 \\
\hline S9 & 4,6 & 19,8 & 13,3 * & 14,7 * & 37,7 * & 34,6 & 12,1 & 2,0 & 7,5 & 0,7 \\
\hline S10 & 2,4 & 24,8 & 12,6 * & 11,5 * & 34,8 * & 26,6 * & 9,3 & 1,4 & 9,3 & 1,0 \\
\hline Total & $5,1 * *$ & 22,5 ** & 18,4 ** & $8,8 * *$ & 30,1 ** & $31,3 * *$ & $10,3 * *$ & $1,6 * *$ & $7,7 * *$ & $1,3 * *$ \\
\hline \multicolumn{11}{|l|}{ Não SUS } \\
\hline NS1 & 6,0 & 14,7 * & 37,3 * & 3,4 & 6,1 * & 85,8 * & 9,3 & 1,2 & 8,8 & 0,5 \\
\hline NS2 & 9,0 & 18,3 & 33,9 & 4,0 & 7,5 & 90,5 * & 8,5 & 1,5 & 9,1 & 0,6 \\
\hline NS3 & 9,8 & 23,0 * & 30,5 * & 6,4 * & 12,1 * & 88,1 * & 7,6 * & 1,1 & 6,6 * & 0,8 \\
\hline NS4 & 9,6 & 19,7 * & 32,6 * & 2,7 & 10,1 * & 85,0 & 8,7 & 1,2 & 8,4 & 0,4 \\
\hline NS5 & 9,8 & 13,7 & 30,8 * & 1,9 * & 6,5 & 87,8 & 9,8 & 0,8 & 8,8 & 0,6 \\
\hline NS6 & 12,3 & 23,5 * & 32,5 * & 4,4 * & 8,6 * & 87,9 * & 8,8 & 0,9 & 8,3 & 0,7 \\
\hline NS7 & 12,2 & 27,7 * & 39,5 * & 4,8 * & 11,7 * & 90,0 * & 8,0 & 1,7 & 7,0 & 0,5 \\
\hline Total & $9,8 * *$ & 16,7 ** & $35,9 * *$ & $3,3 * *$ & 7,2 ** & $86,4 * *$ & $9,1 * *$ & $1,2 * *$ & 8,6 ** & 0,6 * \\
\hline
\end{tabular}

* Diferença significante $(p<0,05)$ entre o perfil dos nascidos vivos dos aglomerados de nascimentos SUS e não SUS comparado com a média do conjunto de aglomerados de nascimentos em hospitais SUS e não SUS;

** Diferença significante $(p<0,05)$ entre o perfil dos nascidos vivos do Município de São Paulo e a média dos aglomerados de nascimentos em hospitais SUS e não SUS.

\section{Discussão}

Os resultados obtidos mostraram existir dependência espacial dos nascimentos SUS e não SUS no Município de São Paulo, a exemplo de estudos anteriores 11,21. Os nascimentos ocorridos em hospitais SUS formaram aglomerados situados em sua maioria nas regiões periféricas da cidade. Ao contrário, os nascimentos em hospitais não SUS formaram aglomerados na região central da cidade. Observou-se também que há características maternas, de gestação e de recém-nascidos distintas nesses aglomerados. Os nascimentos não incluídos nos aglomerados têm distribuição espacial aleatória, sem dependência espacial, aproximando-se da distribuição do Município de São Paulo.

O SUS é uma política pública de saúde que tem, entre suas diretrizes, o acesso universal e gratuito aos serviços de saúde e foi responsável pela ampliação do atendimento ao parto no país. Estudos evidenciam que a rede SUS, especialmente a pública, nas capitais da Região Sudeste do país, apresenta maior complexidade, elevado volume de partos e é equipada com UTI neonatal e adulto 21,22. No presente estudo, a rede privada foi responsável por $40 \%$ dos nascimentos realizados mediante pagamento direto ou por meio de planos de saúde, sendo que a maior parte dos planos são benefícios indiretos vinculados à inserção ao mercado formal de trabalho. A região metropolitana de São Paulo apresenta a maior cobertura populacional dos planos de saúde privados do país, e a rede SUS, responsável por $60 \%$ dos nascimentos, continua sendo a maior responsável por esse atendimento no Município de São Paulo. 
A técnica SaTScan permitiu identificar a existência de dez aglomerados SUS, sendo oito aglomerados situados nas bordas do município e dois (S7 e S10) localizados em áreas mais centrais, em um ambiente deteriorado, com forte presença de residências precárias, como cortiços 23, e imigrantes recentes 24 . Os nascimentos não SUS formaram sete aglomerados contíguos localizados na região central da cidade. Os resultados obtidos são consistentes com vários estudos que mostram que o território do Município de São Paulo apresenta uma segregação espacial radial-concêntrica marcada pela diferenciação socioeconômica e pelas condições de vida e saúde, na medida em que se afasta do centro em direção às periferias 8,11,25,26,27,28.

Os aglomerados de nascimentos SUS em seu conjunto estavam localizados em áreas mais periféricas do Município de São Paulo, com elevada frequência de domicílios subnormais e de baixa renda. As diferenças observadas quanto às características espaciais e socioeconômicas dos territórios refletem-se nas características maternas. Verificaram-se significativos diferenciais de escolaridade e idade com os aglomerados de nascimentos SUS, com maior frequência de mães adolescentes e de baixa escolaridade.

Nossos resultados vão na mesma direção de estudos anteriores 11,29. Um deles, realizado em Belo Horizonte (Minas Gerais), no ano de 2001, encontrou autocorrelação espacial significativa em relação às características maternas dos nascimentos 29. Outro, realizado no Município de São Paulo, com uma amostra de nascimentos, empregando a técnica de kernel, identificou áreas de concentração de nascimentos SUS nas regiões periféricas e nascimentos não SUS na região central 11, além de diferenciais socioeconômicos maternos. No entanto, os aglomerados SUS e não SUS não são homogêneos entre si, à semelhança do observado com relação à distribuição espacial da privação na Região Metropolitana de São Paulo 30. Um dos aglomerados SUS (S9) situado na região sudeste é praticamente formado pelos nascimentos de mães da favela de Heliópolis, porém essa característica se diferencia do grande aglomerado localizado na região leste da cidade, que apresenta pequena proporção de favelas, mas essa é uma área onde há predominância de residências autoconstruídas, que também é uma das características da pobreza da Região Metropolitana de São Paulo 30. Torres et al. 30 apontam que os investimentos em políticas sociais, que visavam a reduzir as desigualdades socioespaciais da cidade, nem sempre foram efetivos e levaram a uma heterogeneidade da pobreza.

O mapeamento dos aglomerados identificou que áreas com maior proporção de domicílios de baixa renda apresentam maior número de mães adolescentes, de baixa escolaridade e de pré-natal inadequado, tanto nos aglomerados de nascimento SUS quanto nos não SUS. Estudo sobre os diferenciais intraurbanos dos nascimentos no Município de São Paulo 8 mostrou existir uma associação entre vulnerabilidade social e proporção de mães com menos de 18 anos, baixa escolaridade materna e menor número de consultas de pré-natal. Também foi identificada a existência de correlação espacial entre proporção de mães adolescentes e situação socioeconômica em nascimentos no Município de São Carlos (São Paulo) 31. Estudo realizado na região metropolitana de São Paulo mostrou que havia importantes diferenciais de fecundidade segundo vulnerabilidade social 32.

Com relação à idade materna, observa-se que, nos aglomerados SUS, há uma maior proporção de mães adolescentes, ao passo que nos aglomerados não SUS predominam mães com 35 anos e mais. São resultados semelhantes aos encontrados por outros autores, em estudos realizados com puérperas de hospitais públicos e privados no Município do São Paulo 11,33. A taxa de fecundidade total (TFT) tem mostrado tendência de declínio no Município de São Paulo, entre 2000 e 2010, com uma mudança na estrutura etária das mulheres. Houve um aumento da taxa específica de fecundidade das mulheres com trinta anos e mais e uma redução da taxa específica das mulheres mais jovens 34 . Outro estudo mostrou que houve um pequeno declínio da proporção de nascimentos de mães adolescentes no Município de São Paulo e um aumento mais expressivo da proporção de nascimentos de mães de 35 anos e mais ${ }^{35}$. Nossos resultados são concordantes com estudos que mostram existir diferenças na estrutura etária das mulheres residentes nos distritos administrativos periféricos e centrais do Município de São Paulo 32.

Um dos indicadores de qualidade do Programa Rede Cegonha 36 do Ministério da Saúde é a realização de mais de sete consultas de pré-natal, dado que o Programa de Humanização no Pré-natal e Nascimento 37 recomenda a realização de, no mínimo, seis consultas de pré-natal. Ainda que se observe um crescimento da assistência pré-natal 38,39, continuam presentes diferenciais marcantes. A 
proporção de mães com número de consultas inferiores ao recomendado era quatro vezes maior entre as mães dos aglomerados SUS que dos não SUS. Os aglomerados SUS com alta proporção de pré-natal inadequado, territórios com baixa renda, também apresentaram maior proporção de adolescentes e de mães com baixa escolaridade. Os estudos de Gama et al. 40 e Cesar et al. 41 mostraram que características como mães adolescentes, baixa escolaridade e baixa renda estão associadas à realização de menor número de consultas de pré-natal.

A diferença entre os aglomerados SUS e não SUS em relação à proporção de cesáreas é compatível com o encontrado em outras pesquisas 29,41,42,43. Estudo com dados da pesquisa Nascer no Brasil mostrou que, nos hospitais privados, as taxas de cesárea são mais elevadas $(87,9 \%)$ do que nos hospitais públicos (42,9\%) 42. Estudo realizado no Rio Grande do Sul em 200741 também mostrou existir elevado diferencial da proporção de cesáreas realizadas em hospitais privados (70\%) em relação aos hospitais públicos (43\%). Estudo realizado em Belo Horizonte 29 verificou que a taxa de cesárea variava de $28 \%$ a $67 \%$, sendo que as maiores taxas foram encontradas nas regiões de melhor condição socioeconômica. Nossos resultados mostraram que, nos aglomerados, os diferenciais de taxas de cesáreas entre os nascimentos em hospitais SUS e privados são ainda mais acentuados.

No conjunto dos aglomerados SUS, foi observada uma maior proporção estatisticamente significante de nascimentos com baixo peso, se comparada à dos aglomerados não SUS. Esse resultado alinha-se ao obtido em estudo no Município de São Paulo 8 que encontrou um gradiente de baixo peso ao nascer conforme aumenta a vulnerabilidade social. Foi identificada uma proporção mais elevada de nascimentos pré-termos nos aglomerados SUS que nos não SUS, verificando-se ainda que, nos aglomerados não SUS, há uma proporção mais elevada de pré-termos tardios (32 a 36 semanas), ao passo que, nos aglomerados SUS, há maior proporção de nascimentos pré-termos precoces $(<32$ semanas). Esses resultados são próximos aos encontrados em estudo realizado no Município de São Paulo 44, em que os autores sugerem que a elevada proporção de cesáreas eletivas em hospitais não SUS contribui para a maior proporção de pré-termos tardios (34 a 36 semanas), ao passo que, na rede SUS, condições socioeconômicas desfavoráveis podem estar associadas a partos espontâneos prematuros e ao pré-natal inadequado. Entretanto, por se tratar de dados secundários, não há como avaliar se a não realização do mínimo de consultas recomendado foi devido ao parto prematuro, por falha na assistência ou devido a fatores sociodemográficos que dificultam o acesso ao pré-natal. Outras características maternas também estão associadas à prematuridade, como baixa escolaridade, mães adolescentes ou com idade igual ou acima de 35 anos 45 .

Verificou-se também que a distância entre a residência materna e o hospital, para os nascimentos nos aglomerados SUS, era menor que aquela observada nos aglomerados não SUS, indicando provável efeito do programa Rede de Proteção à Mãe Paulistana, implementado no Município de São Paulo em 2006, e que visa a garantir o acesso às ações e a serviços de promoção, prevenção e assistência à saúde materno-infantil, do pré-natal ao puerpério 46. Uma das ações desse programa é a rede de referência de maternidade, de modo a regionalizar os partos de acordo com o endereço e a classificação de risco gestacional. Ainda que o cálculo da distância em linha reta não considere a existência de barreiras naturais ou de mobilidade urbana, pode servir de referência de facilidade de acesso na atenção ao parto.

Por outro lado, embora os aglomerados não SUS estejam concentrados na área central onde há concentração dos equipamentos de saúde privada, a distância média entre a residência materna e os hospitais foi maior que aquela dos nascimentos SUS, sendo que, em dois aglomerados mais próximos da periferia, as distâncias indicam que as mães residentes daquelas áreas tiveram os maiores deslocamentos observados no estudo. Tal fato pode ser explicado porque, nos planos privados de saúde, não há a preocupação com regionalização, e nem sempre o serviço disponível à condição do plano está perto da residência. Ainda com respeito a esse ponto, alguns estudos mostram que há relação entre distância do serviço de saúde e resultados adversos, tanto para a mãe como para o recém-nascido, aumento de intervenções no parto e de mortalidade neonatal 47,48.

O estudo foi realizado em ano censitário, com disponibilidade de informações para pequenas unidades territoriais (setores censitários), o que permitiu o emprego de técnicas de análise espacial. $\mathrm{O}$ SaTScan, utilizado nesse estudo para detecção dos aglomerados de nascidos vivos, é muito usado para detecção de padrões geográficos de doenças infecciosas. Porém, poucos foram os estudos encontrados 
para análise de nascimentos e eventos materno-infantis 49, o que não permite que se avalie a eficiência desse instrumento para esse tipo de análise. No entanto, dada a plausibilidade dos resultados, pode-se inferir que se trata de uma ferramenta útil para detecção de aglomerados de nascimentos.

Este é um estudo realizado com dados secundários e podem ocorrer erros de registros de dados. Cerca de 5\% dos nascimentos vivos não foram passíveis de geocodificação, devido ao registro do endereço que não permitia sua localização, fato que é mais frequente em loteamentos recentes, na maioria das vezes localizados na periferia da cidade, o que pode ter minimizado parte dos diferenciais socioeconômicos encontrados. A identificação dos nascimentos em SUS e não SUS, segundo o hospital do parto e não individualmente para cada nascimento estudado, pode se tornar um viés, dado que os hospitais mistos que atendem a usuários SUS e não SUS foram considerados como SUS, minimizando os diferenciais socioeconômicos.

\section{Conclusão}

A formação de aglomerados de nascimentos SUS e não SUS mostra que há diferenciais das características maternas, de gestação, de parto e de recém-nascidos no Município de São Paulo. Os aglomerados de nascimentos SUS e não SUS apresentam distribuição espacial radial-concêntrica no Município de São Paulo, indicando que os nascimentos apresentam os mesmos diferenciais socioeconômicos existentes na cidade. Entretanto, há uma heterogeneidade interna dos aglomerados SUS e não SUS. A menor distância encontrada entre a residência materna e o hospital de nascimento mostra a regionalização da atenção ao parto no SUS no Município de São Paulo. A detecção de aglomerados e a caracterização socioeconômica das áreas contribuem para o entendimento do padrão espacial de nascimentos e intervenções de saúde pública.

\section{Colaboradores}

P. C. Santos participou do delineamento do estudo, da análise dos dados e da redação do trabalho. Z. P. Silva e M. F. Almeida participaram do delineamento do estudo, da redação do trabalho e da revisão crítica. F. Chiaravalloti Neto e G. P. Alencar participaram do delineamento do estudo.

\section{Agradecimentos}

Este trabalho faz parte do projeto Distribuição Espacial dos Nascimentos, Trajeto das Mães dos Domicílios aos Hospitais de Parto e Mortalidade Neonatal no Município de São Paulo, 2010, que recebeu financiamento da Fundação de Amparo à Pesquisa do Estado de São Paulo (Fapesp; processo no 2011/21718-1). P. C. Santos é bolsista do Conselho Nacional de Desenvolvimento Científico e Tecnológico (CNPq). 


\section{Referências}

1. Barcellos C, Sabroza PC, Peiter P, Rojas LI. Organização espacial, saúde e qualidade de vida: a análise espacial e o uso de indicadores na avaliação de situações de saúde. Inf Epidemiol SUS 2002; 11:129-38.

2. Krieger N, Chen J, Waterman P, Soobader M, Subramanian S, Carson R. Choosing area based socioeconomic measures to monitor social inequalities in low birth weight and childhood lead poisoning: The Public Health Disparities Geocoding Project (US). J Epidemiol Community Health 2003; 57:186-99.

3. Susser M, Susser E. Choosing a future for epidemiology: I. Eras and paradigms. Am J Public Health 1996; 86:668-73.

4. d'Orsi E, Carvalho MS, Cruz OG. Similarity between neonatal profile and socioeconomic index: a spatial approach. Cad Saúde Pública 2005; 21:786-94.

5. Nascimento LFC, Batista GT, Dias NW, Catelani CS, Becker D, Rodrigues L. Análise espacial da mortalidade neonatal no Vale do Paraíba, 1999 a 2001. Rev Saúde Pública 2007; 41:94-100.

6. Nogueira MJ, Silva BFA, Barcelos SM, Schall VT. Análise da distribuição espacial da gravidez adolescente no Município de Belo Horizonte - MG. Rev Bras Epidemiol 2009; 12:297-312.

7. Barcellos C, Acosta LMW, Lisboa E, Bastos FI. Surveillance of mother-to-child HIV transmission: socioeconomic and health care coverage indicators. Rev Saúde Pública 2009; 43:100614.

8. Minuci EG, Almeida MF. Diferenciais intra -urbanos de peso ao nascer no município de São Paulo. Rev Saúde Pública 2009; 43:256-66.

9. Predebon KM, Mathias TAF, Aidar T, Rodrigues AL. Desigualdade sócio-espacial expressa por indicadores do Sistema de Informações sobre Nascidos Vivos (SINASC). Cad Saúde Pública 2010; 26:1583-94.

10. Melo EC, Mathias TAF. Spatial distribution and self-correlation of mother and child health indicators in the state of Parana, Brazil. Rev Latinoam Enferm 2010; 18:1177-86.

11. Santos PC, Silva ZP, Chiaravalloti Neto F, Almeida MF. Análise espacial dos aglomerados de nascimentos ocorridos em hospitais SUS e não SUS do município de São Paulo, Brasil. Ciênc Saúde Coletiva 2014; 19:235-44.

12. Almeida WS, Szwarcwald CL. Mortalidade infantil e acesso geográfico ao parto nos municípios brasileiros. Rev Saúde Pública 2012; 46:68-76.

13. Souza GOC. Área de abrangência de atendimento dos hospitais e construção de suas bases territoriais na Região Metropolitana de São Paulo. São Paulo Perspect 2008; 22:72-86.
14. Cardim LL, Ferraudo AS, Pacheco STA, Reis RB, Silva MMN, Carneiro DDMT, et al. Análises espaciais na identificação das áreas de risco para a esquistossomose mansônica no Município de Lauro de Freitas, Bahia, Brasil. Cad Saúde Pública 2011; 27:899-908.

15. Kulldorff M. SaTScanTM user guide, 2015. http://www.satscan.org/techdoc.html (acessado em 24/Ago/2016).

16. Caiado MCS. Deslocamentos intra-urbanos e estruturação socioespacial na metrópole brasiliense. São Paulo Perspect 2005; 19:64-77.

17. Penchansky DBA, Thomas JW. The concept of access - definition and relationship to consumer satisfaction. Med Care 1981; 19:127-40.

18. Barcellos C, Santos SM. Colocando dados no mapa: a escolha da unidade espacial de agregação e integração de bases de dados em saúde e ambiente através do geoprocessamento. Inf Epidemiol SUS 1997; 6:21-9.

19 Barcellos C, Bastos FI. Geoprocessamento, ambiente e saúde: uma união possível? Cad Saúde Pública 1996; 12:389-97.

20. Kulldorff M, Nagarwalla N. Spatial disease clusters: detection and inference. Stat Med 1995; 14:799-810.

21. Bittencourt SDA, Reis LGC, Ramos MM, Rattner D, Rodrigues PL, Neves DCO, et al. Estrutura das maternidades: aspectos relevantes para a qualidade da atenção ao parto e nascimento. Cad Saúde Pública 2014; 30 Suppl 1:S208-19.

22. Coordenação de Epidemiologia e Informação, Secretaria Municipal da Saúde. Nascer na cidade de São Paulo: 15 anos do SINASC. São Paulo: Secretaria Municipal da Saúde; 2015. (Boletim CEInfo Análise).

23. Piccini A. Cortiços em São Paulo: conceito e preconceito na reestruturação do centro urbano de São Paulo. São Paulo: Annablume; 1999.

24. Coordenação de Epidemiologia e Informação, Secretaria Municipal da Saúde. Alguns aspectos da saúde de imigrantes e refugiados recentes no município de São Paulo. São Paulo: Secretaria Municipal da Saúde; 2015. (Boletim CEInfo Análise).

25. Monteiro CA, Benício MHA, Baldijão MFA. Mortalidade no primeiro ano de vida e a distribuição de renda e de recursos públicos de saúde, São Paulo (Brasil). Rev Saúde Pública 1980; 14:515-39.

26. Taschner SP, Bógus L. A cidade dos anéis: São Paulo. In: Queiroz LC, organizador. O futuro das metrópoles: desigualdades e governabilidade. Rio de Janeiro: Revan/Fase; 2000. p. 247-84. 
27. Villaça F. Efeitos do espaço sobre o social na metrópole brasileira. In: Souza MA, organizador. Metrópole e globalização: conhecendo a cidade de São Paulo. São Paulo: Cedesp; 1999. p. 221-36.

28. Bonduki N, Rolnik R. Periferia da Grande São Paulo: reprodução do espaço como expediente de reprodução da força de trabalho. In: Maricato E, organizador. A produção capitalista da casa (e da cidade) do Brasil industrial. São Paulo: Alfa-Ômega; 1982. p. 17-154.

29. Friche AAL, Caiaffa WT, César CC, Goulart LMF, Almeida MCM. Indicadores de saúde materno infantil em Belo Horizonte, Minas Gerais, Brasil, 2001: análise dos diferenciais intra-urbanos. Cad Saúde Pública 2006; 22:1955-65.

30. Torres HG, Marques E, Ferreira MP, Bitar S. Pobreza e espaço: padrões de segregação em São Paulo. Estud Av 2003; 17:97-128.

31. Ferreira RA, Ferriani MGC, Mello DF, Carvalho IP, Cano MA, Oliveira LA. Análise espacial da vulnerabilidade social da gravidez na adolescência. Cad Saúde Pública 2012; 28:313-23.

32. Brito ALS, Yazaki LM, Maia PB. Vulnerabilidade ao nascer no espaço metropolitano. São Paulo Perspect 2006; 20:18-32.

33. Silva ZP, Almeida MF, Ortiz LP, Alencar GP, Alencar AP, Schoeps D, et al. Morte neonatal precoce segundo complexidade hospitalar e rede SUS e não SUS na Região Metropolitana de São Paulo, Brasil. Cad Saúde Pública 2010; 26:123-34.

34. Yazaki LM. Diferenciais regionais de fecundidade no Município de São Paulo. São Paulo Demográfico 2016; 16(2).

35. Coordenação de Epidemiologia e Informação, Secretaria Municipal da Saúde. Gestação na adolescência no município de São Paulo. São Paulo: Secretaria Municipal da Saúde; 2015. (Boletim Eletrônico CEInfo, ano 6, n. 2).

36. Ministério da Saúde. Portaria no 1.459 , de 24 de junho de 2011. Institui, no âmbito do Sistema Único de Saúde - SUS - a Rede Cegonha. Diário Oficial da União 2011; 27 jun.

37. Ministério da Saúde. Programa de Humanização no Pré-Natal e Nascimento. Brasília: Ministério da Saúde; 2002.

38. Alencar GP, Silva ZP, Santos PC, Raspantini PR, Moura BLA, Almeida MF, et al. What is the impact of interventions that prevent fetal mortality on the increase of preterm live births in the State of Sao Paulo, Brazil? BMC Pregnancy Childbirth 2015; 15:152.

39. Viellas EF, Domingues RMSM, Dias MAB, Gama SGN, Theme Filha MM, Costa JV, et al. Assistência pré-natal no Brasil. Cad Saúde Pública 2014; 30 Suppl 1:S85-100.
40. Gama SG, Szwarcwald CL, Sabroza AR, Branco VC, Leal MC. Fatores associados à assistência pré-natal precária em uma amostra de puérperas adolescentes em maternidades do Município do Rio de Janeiro, 1999-2000. Cad Saúde Pública 2004; 20 Suppl 1:S101-11.

41. Cesar JA, Mano OS, Carlotto K, GonzalesChica DA, Mendoza-Sassi RA. Público versus privado: avaliando a assistência à gestação e ao parto no extremo sul do Brasil. Rev Bras Saúde Matern Infant 2011; 11:257-63.

42. Rebelo F, Da Rocha CMM, Cortes TR, Dutra CL, Kac G. High cesarean prevalence in a national population-based study in Brazil: the role of private practice. Acta Obstet Gynecol Scand 2010; 89:903-8.

43. Nakamura-Pereira M, Leal MC, EstevesPereira AP, Domingues RMS, Torres JA, Moreira ME, et al. Use of Robson classification to assess cesarean section rate in Brazil: the role of source of payment for childbirth. Reprod Health 2016; 13 Suppl 3:128.

44. Raspantini PR, Miranda MJ, Silva ZP, Alencar GP, Diniz SG, Almeida MF. O impacto do tipo de hospital e tipo de parto sobre a idade gestacional ao nascer no Município de São Paulo, 2013-2014. Rev Bras Epidemiol 2016; 19:87882.

45. Tedesco RP, Passini Jr. R, Cecatti JG, Camargo RS, Pacagnella RC, Sousa MH. Estimation of preterm birth rate, associated factors and maternal morbidity from a demographic and health survey in Brazil. Matern Child Health J 2013; 17:1638-47.

46. Secretaria Municipal de Saúde de São Paulo. Decreto Municipal no 46.966/2006. Regulamenta a Lei no $13.211 / 2001$, estruturando a Rede de Proteção à Mãe Paulistana, para a gestão e execução da rede de serviços de saúde de assistência obstétrica e neonatal no Município de São Paulo. Diário Oficial da Cidade de São Paulo 2006; 3 fev.

47. Ravelli A, Jager K, De Groot M, Erwich J, Rijninks-Van Driel G, Tromp M, et al. Travel time from home to hospital and adverse perinatal outcomes in women at term in the Netherlands. BJOG 2011; 118:457-65.

48. Grzybowski S, Stoll K, Kornelsen J. Distance matters: a population based study examining access to maternity services for rural women. BMC Health Serv Res 2011; 11:147.

49. SaTScan. SaTScan bibliography. http://www. satscan.org/references.html (acessado em 24/ Ago/2016). 


\section{Abstract}

This study aimed to identify birth clusters according to type of hospital (SUS vs. non-SUS) and the existence of differences in socioeconomic, maternal, neonatal, and healthcare access characteristics, measured by the distance between the mothers' homes and the hospitals where they gave birth. Births to mothers residing in the city of São Paulo, Brazil, in 2010 were georeferenced and allocated in 310 weighting areas from the population census, in addition to classifying them according to hospital of birth (SUS vs. non-SUS). Spatial clusters were identified through the spatial sweep technique for spatial dependence of SUS and non-SUS births, leading to the formation of ten SUS clusters and seven non-SUS clusters. Births in non-SUS hospitals formed clusters in the city's central area, with a lower proportion of low-income households. The SUS birth clusters were located on the outskirts of the city, where there are more households in subnormal clusters. Both SUS and non-SUS clusters were not internally homogeneous, showing differences in maternal age, schooling, and number of prenatal visits and very premature newborns. The theoretical mean distance traveled by mothers to the hospital was $51.8 \%$ lower in the SUS clusters $(5.1 \mathrm{~km})$ than in the non-SUS clusters $(9.8 \mathrm{~km})$. The formation of birth clusters showed differences in maternal, pregnancy, childbirth, and neonatal characteristics, in addition to displaying a radialconcentric spatial distribution, reflecting the city's prevailing socioeconomic differences. The shorter distance in SUS births indicates regionalization of childbirth care in the city of São Paulo.

Live Birth; Health Status Disparities; Geographic Information Systems; Public Hospitals;

Private Hospitals

\section{Resumen}

El objetivo del estudio fue identificar aglomerados de nacimientos, según el tipo de hospital (SUS y no SUS), y la existencia de diferenciales en cuanto a características socioeconómicas, materno-infantiles y de acceso, calculados por la distancia entre las residencias maternas y los hospitales donde se produjeron los partos. Los nacimientos que se produjeron con madres residentes en el municipio de São Paulo, Brasil, en 2010 fueron georreferenciados y asignados a las 310 áreas de ponderación del censo demográfico, además de clasificados según el hospital de nacimiento (SUS y no SUS). Se identificaron aglomerados espaciales mediante la técnica de barrido espacial para la dependencia espacial de los nacimientos SUS y no SUS, formando diez aglomerados SUS y siete no SUS. Los nacimientos en hospitales no SUS constituyeron aglomerados, situados en el área central, donde existe una menor proporción de domicilios de baja renta. Los aglomerados de nacidos vivos SUS estaban ubicados en el extrarradio de la ciudad, donde son más frecuentes domicilios en aglomerados por debajo de los estándares normales. Los aglomerados tanto SUS, como no SUS, no son homogéneos entre sí, debido a que existen diferencias referentes a la edad de las madres, escolaridad, número de consultas prenatales y recién nacidos muy prematuros. La distancia media teórica recorrida por las madres hasta el hospital fue un 51,8\% menor en los aglomerados SUS $(5,1 \mathrm{~km})$ que en los no SUS (9,8km). La formación de aglomerados de nacimientos mostró diferenciales en las características maternas, gestación, parto y recién nacidos, además de haber presentado distribución espacial radial-concéntrica, reflejando los diferenciales socioeconómicos existentes en la ciudad. La menor distancia en los nacimientos SUS indica la regionalización de la asistencia al parto en el municipio de São Paulo.

Nacimiento Vivo; Disparidades en el Estado de Salud; Sistemas de Información Geográfica; Hospitales Públicos; Hospitales Privados
Recebido em 09/Set/2016

Versão final representada em 07/Ago/2017

Aprovado em 04/Dez/2017 